\title{
Utilização de consulta médica e hipertensão arterial sistêmica nas áreas urbanas e rurais do Brasil, segundo dados da PNAD 2008
}

\author{
Use of medical consultations and the occurrence of systemic \\ arterial hypertension in urban and rural areas of Brazil, \\ according to PNAD data 2008
}

Jessica Pronestino de Lima Moreira ${ }^{1}$

José Rodrigo de Moraes ${ }^{2}$

Ronir Raggio Luiz ${ }^{1}$

\footnotetext{
${ }^{1}$ Instituto de Estudos em Saúde Coletiva, Universidade Federal do Rio de Janeiro. Avenida Horácio Macedo S/N, Ilha do Fundão. 21941-598 Rio de Janeiro RJ. jessica@iesc.ufrj.br ${ }^{2}$ Universidade Federal Fluminense
}

\begin{abstract}
The use of medical consultations is influenced by determinants such as healthcare needs and service characteristics, which depend on whether the environment is urban or rural. The scope was to estimate the proportions of individuals attending medical consultations over the previous 12 months with and without self-reported systemic arterial hypertension (SAH) living in urban and rural areas, and to analyze the patterns of consultation use and associations. This was a sectional study, using PNAD 2008. Logistic regression was performed to obtain crude and adjusted odds ratios (ORs), according to self-reported SAH and household situation. 70.6\% of adult Brazilians consult physicians. The association between the presence of SAH and attending medical consultations was 3.63 (OR) times greater in urban areas. The incidence of consultation with physicians was greater among women, individuals using continuous medication or who had health insurance plans or funding for the last consultation, people who reported a disease or restriction in mobility and those with self-reported poor health, in all strata. Multivariate analysis modified the associations of all variables. The differences between the two areas suggested that access policies need to be implemented, with the aim of reducing inequalities.
\end{abstract}

Key words Health service accessibility, Hypertension, Urban population
Resumo A utilização de consultas médicas é influenciada por determinantes, como necessidades de saúde e características dos serviços, que dependem se o ambiente é urbano ou rural. Objetiva-se estimar a proporção de consulta ao médico nos últimos 12 meses dos indivíduos que referiram ou não hipertensão arterial sistêmica (HAS), residentes em área urbana e rural, e analisar os padrões de utilização de consulta e associações. Trata-se de um estudo seccional, utilizando a PNAD 2008. Executaram-se regressões logísticas para obter odds ratios (OR's), brutas e ajustadas, por HAS autorreferida e situação do domicílio. Consultaram o médico 70,6\% dos adultos brasileiros. Foi encontrada uma associação entre HAS e consulta ao médico de 3,63 (OR) maior na área urbana. A chance de consulta ao médico foi maior entre as mulheres, os que utilizam medicamentos contínuos, os que possuem plano de saúde ou tiveram financiamento no último atendimento, os que referiram alguma morbidade ou limitação na mobilidade e entre os que referiram estado de saúde ruim, em todos os estratos. A análise multivariada modificou as associações de todas as variáveis. Diferenças nas duas áreas sugerem que políticas de acesso devem ser implantadas, com o objetivo de reduzir iniquidades.

Palavras-chave Acesso aos serviços de saúde, Hipertensão, População urbana 


\section{Introdução}

No campo da Saúde Pública, acesso é um conceito que expressa o grau de ajuste entre as necessidades dos usuários e a oferta de serviços ${ }^{1,2}$. Em outras palavras, está relacionado ao perfil das necessidades de um determinado grupo populacional, à conversão destas necessidades em demanda e à transformação da demanda em utilização de serviços de saúde ${ }^{3-5}$.

Segundo Hulka et al. ${ }^{6}$, os determinantes da utilização dos serviços de saúde podem ser descritos como aqueles fatores relacionados: (i) à necessidade de saúde; (ii) às características demográficas; (iii) à disponibilidade de profissionais de saúde; (iv) às características de organização dos serviços de saúde; e (v) ao mecanismo de financiamento. Diante destes determinantes, pode-se esperar um diferencial na utilização de serviços sob diferentes condições, em particular nos ambientes urbano e rural. De acordo com Travassos e Viacava ${ }^{7}$, em geral, é observado menor acesso e conseqüente menor utilização de serviços de saúde nas populações rurais. Além da menor disponibilidade de serviços, grandes distâncias a serem percorridas, dificuldades de transporte e baixa renda são fatores que, associados, reduzem a utilização de serviços de saúde nestes ambientes ${ }^{7}$.

Acrescente-se ainda que, de modo geral, os portadores de doenças crônicas, em virtude da necessidade de controle, colaboram para elevar a demanda e utilização dos serviços de saúde do país ${ }^{8}$. A hipertensão arterial sistêmica (HAS), por exemplo, considerada concomitantemente uma doença e um fator de risco para doenças cardiovasculares, com uma prevalência elevada - estimada em aproximadamente $20 \%$ da população brasileira - contribui bastante para este aumento das necessidades de saúde do país. A HAS exige uma complexidade de recursos necessários para seu controle, como serviços médicos, pessoal treinado e medicamentos. Além disso, esta enfermidade é geralmente desconhecida pela metade dos portadores e, entre aqueles que conhecem seu problema, somente a metade deles recebe algum tipo de assistência médica para seu controle ${ }^{9}$. Desta forma, a utilização da hipertensão autorreferida, em que o agravo é mensurado por meio de entrevista, pode subestimar a verdadeira prevalência na população, embora vários estudos sugiram sua validade para grandes populações ${ }^{10-13}$.

No Brasil, o Instituto Brasileiro de Geografia e Estatística (IBGE) realiza a Pesquisa Nacional por Amostra de Domicílios (PNAD), que possui informações sobre morbidades crônicas autorreferidas, além de fatores que permitem estudos de acesso e utilização dos serviços de saúde. Um indicador de utilização aos serviços de saúde, disponível na PNAD e frequentemente utilizado, se refere às consultas médicas realizadas anualmente.

Este artigo tem como objetivo estimar a proporção de consulta ao médico nos últimos 12 meses dos indivíduos que referiram e dos que não referiram HAS, residentes em área urbana e rural, e analisar os padrões de utilização de consulta médica e as possíveis associações, utilizando dados recentes e representativos da população brasileira, obtidos através da PNAD, realizada no ano de 2008.

\section{Material e métodos}

\section{População de estudo}

Foi utilizado como base de dados neste estudo o inquérito populacional de abrangência nacional, denominada Pesquisa Nacional por Amostra de Domicílios (PNAD), realizada pelo Instituto Brasileiro de Geografia e Estatística (IBGE) no ano de 2008, bem como seu suplemento de saúde. O Suplemento de Saúde da PNAD é um questionário adicional realizado quinquenalmente e que contém várias informações sobre saúde, incluindo acesso e utilização dos serviços. Dos 391.868 indivíduos investigados na PNAD neste ano, foram selecionados para o presente estudo os adultos, com 20 anos ou mais, que correspondem a 257.816 indivíduos. Foram excluídos da amostra indivíduos que apresentaram dados faltantes em alguma variável, totalizando 256.583 indivíduos considerados.

A PNAD utiliza-se de um desenho de amostragem denominado complexo, que consiste na agregação de métodos de amostragem clássicos (estratificação e conglomeração), com probabilidades desiguais de seleção em dois ou três estágios, que dependem se municípios selecionados são classificados como autorrepresentativos ou não. Os municípios situados em Regiões Metropolitanas ou em unidades da federação sem região metropolitana, porém grandes em termos populacionais, são chamados de municípios autorrepresentativos. Os municípios não autorrepresentativos são aqueles situados fora das Regiões Metropolitanas ou considerados não tão grandes em termos populacionais ${ }^{14}$. Em Silva et al. ${ }^{15}$ e Lee et al. ${ }^{16}$ podem ser obtidas maiores informações sobre o planejamento amostral complexo. 


\section{Variáveis estudadas}

A variável consultou o médico nos últimos 12 meses foi considerada como desfecho no presente estudo. Optou-se por utilizar as variáveis $h i$ pertensão autorreferida e situação do domicílio como variáveis de estratificação, apresentando os resultados em separado para os hipertensos e não hipertensos autorreferidos, assim como para área de localização do domicílio (urbana ou rural). Foram consideradas também outras variáveis disponíveis na PNAD e que podem estar relacionadas com o desfecho. São elas: região geográfica, sexo, faixa etária, cor/raça, anos de estudo, renda familiar per capita, autopercepção de saúde, mobilidade física, morbidade autorreferida, medicamentos de uso contínuo, direito a plano de saúde, financiamento do último atendimento.

A situação do domicílio (urbana e rural) foi definida segundo o IBGE ${ }^{14}$, de modo que a situação urbana agregou 3 categorias: 1) Urbana - Cidade ou vila, área urbanizada, 2) Urbana - Cidade ou vila, área não urbanizada e 3) Urbana - Área urbana isolada. Para definir a situação rural juntaram-se 5 categorias relativas a esta área: 1) $R u$ ral - Aglomerado rural de extensão urbana, 2) $R u$ ral-Aglomerado rural, isolado, povoado, 3) Rural - Aglomerado rural, isolado, núcleo, 4) Rural Aglomerado rural, isolado, outros aglomerados e 5) Rural - Zona rural exclusive aglomerado rural.

A mobilidade física foi classificada em três níveis: limitada, pouco limitada e sem limitação. Considerou-se como limitada quando os indivíduos não conseguem ou têm grande dificuldade para realizar pelo menos uma das 8 características de mobilidade física levantadas pela PNAD; pouco limitada quando possuem pequena dificuldade em pelo menos uma das características de mobilidade e sem limitação quando não apresentam nenhum grau de dificuldade nas características de mobilidade. As 8 características de mobilidade da PNAD são: 1) Alimentar-se, tomar banho ou ir ao banheiro; 2) Correr, levantar objetos pesados, praticar esporte ou realizar trabalhos pesados; 3) Empurrar mesa ou realizar consertos domésticos; 4) Subir ladeira ou escada; 5) Abaixar-se, ajoelhar-se ou curvar-se; 6) Andar mais do que $1 \mathrm{~km}$; 7) Andar cerca de 100 metros; 8) Fazer compras de alimentos, roupas e medicamentos, sem ajuda.

A variável morbidade autorreferida foi definida como o indivíduo que referiu pelo menos 1 das doenças, que foram agrupadas em 3 categorias da seguinte forma: 1) Diabetes ou doença do coração ou insuficiência renal crônica; 2) Outra doença (doença de coluna ou costas, artrite ou reumatismo, câncer, bronquite ou asma, depressão, tuberculose, tendinite ou tenossinovite e cirrose); e 3) Sem morbidade. Tendo em vista que a morbidade HAS se configura como variável de estratificação neste estudo, esta não foi considerada para a construção da variável morbidade autorreferida.

A variável financiamento do último atendimento refere-se àquele ocorrido nas duas semanas anteriores à pesquisa. Foram consideradas 5 categorias 1) pagou, para quem efetuou o pagamento através de recursos próprios, 2) plano de saúde, 3) Sistema Único de Saúde (SUS), 4) não procurou ou não foi atendido e 5) sem declaração.

\section{Análise estatística}

As análises foram feitas utilizando o software estatístico SPSS for Windows, versão $17.0^{17}$. Foram incorporadas as informações do plano amostral: estrato, unidade primária de amostragem e peso amostral. O peso amostral foi relativizado, passando a ser utilizada a razão entre o peso definido no desenho da amostra e o peso médio. A utilização do peso relativo mantém o tamanho da amostra e a precisão dos estimadores. Ignorar o desenho de amostragem complexo pode produzir incorreções, comprometendo tanto as estimativas pontuais quanto as suas medidas de precisão e, com isso, as conclusões da pesquisa ${ }^{18}$.

Estimou-se a proporção de indivíduos adultos, com 20 anos ou mais, hipertensos e não hipertensos, que consultaram o médico nos últimos 12 meses, por situação do domicílio. Foi feita a frequência relativa das variáveis relacionadas às condições demográficas, socioeconômicas e de saúde da população. A prevalência da situação do domicílio urbano e de consulta ao médico nos últimos 12 meses foi estimada para cada variável. Regressões logísticas univariadas foram executadas, com o objetivo de obter as medidas de associação odds ratio (OR) das variáveis com o desfecho, além dos respectivos intervalos de confiança de 95\%. Com o objetivo de obter valores de OR ajustados, foram executados dois modelos de regressão logística multivariada, um para os hipertensos e outro para os não hipertensos autorreferidos, considerando todas as variáveis e estratificando pela situação do domicílio (urbana e rural), totalizando quatro modelos. Interações foram testadas considerando um nível de significância restrito de 0,01 , tendo em vista o grande tamanho da amostra. 


\section{Resultados}

Observa-se que a proporção de pessoas que moram em área urbana é muito maior $(85,0 \%)$ do que os que moram na área rural. Em torno de $70 \%$ das pessoas com 20 anos ou mais consultaram o médico nos últimos 12 meses. Verifica-se uma proporção mais elevada de consulta médica na área urbana $(71,8 \%$ vs. $63,6 \%)$. Os hipertensos autorreferidos consultaram mais o médico nos últimos 12 meses do que os não hipertensos $(87,6 \%$ vs. $66,1 \%)$ (Tabela 1$)$.

A Tabela 2 apresenta a distribuição de frequência das variáveis demográficas e socioeconômicas, a proporção de indivíduos residentes em área urbana e a associação com consulta ao médico nos últimos 12 meses. Verifica-se maior proporção de mulheres $(52,5 \%)$, de pessoas com 20 a 35 anos (39,8\%), dos que possuem 8 a 14 anos de estudo $(44,5 \%)$ e dos que não têm rendimento ou possuem renda familiar per capita de até 1 salário mínimo (49,8\%). Aproximadamente $81 \%$ das mulheres consultaram o médico nos últimos 12 meses, enquanto que menos de $60 \%$ dos homens utilizaram este serviço. A proporção de consulta ao médico é maior entre os idosos (65 anos ou mais), apresentando uma chance de utilizar este serviço duas vezes maior, com relação aos indivíduos de 20 a 35 anos. Indivíduos com renda familiar per capita maior que 5 salários mínimos mostraram-se mais fortemente associados com consulta médica $(\mathrm{OR}=2,34)$.

As regressões logísticas univariadas (Tabela 3) mostram que os indivíduos que referiram alguma doença têm chance em torno de três vezes de terem consultado ao médico nos últimos 12 meses. Observou-se que quanto melhor a autopercepção de saúde, menor a chance de consulta ao médico $\left(\mathrm{OR}_{\text {muito bom/bom }}=0,22\right.$ e $\left.\mathrm{OR}_{\text {regular }}=0,53\right)$. Com relação à mobilidade física, verificam-se que $67,4 \%$ das pessoas não possuem limitação, entre- tanto, os que possuem alguma limitação apresentam em torno de 2,5 vezes mais chance de ter consultado o médico $(\mathrm{OR}=2,46$ - pouco limitado e OR=2,68 - limitado). Quase totalidade dos indivíduos que utilizam medicamentos de uso contínuo $(91 \%)$ consultaram o médico nos últimos 12 meses, apresentando uma chance de 3,96 a mais do que os que não utilizam. Os que têm direito a plano de saúde apresentam 2,37 vezes mais chance de consulta médica. Os indivíduos que tiveram o último atendimento financiado pelo plano de saúde ou pelo SUS, apresentam chances de terem consultado o médico nos últimos 12 meses maiores em 6 e 3 vezes, respectivamente, com relação àqueles que pagaram pela consulta.

A Tabela 4 apresenta os modelos logísticos univariados para consulta ao médico nos últimos 12 meses, estratificado entre hipertensos e não hipertensos autorreferidos e por situação do domicílio, bem como os OR's e seus respectivos intervalos de confiança de $95 \%$, para fins de comparação com o resultado encontrado no modelo multivariado.

A Tabela 5 apresenta os OR's ajustados e seus respectivos intervalos de confiança de 95\%, obtidos através dos modelos logísticos multivariados para consulta ao médico nos últimos 12 meses, estratificado entre hipertensos e não hipertensos autorreferidos e por situação do domicílio.

De forma geral, observa-se que as variáveis que apresentam medidas de associação mais elevadas são medicamentos de uso contínuo e financiamento do último atendimento em todos os estratos. Indivíduos que utilizam medicamentos de uso contínuo têm chance em torno de 3 vezes mais de ter consultado o médico nos últimos 12 meses e, entre os não hipertensos da área rural, esta chance aumenta para 3,52. Indivíduos que tiveram o financiamento do último atendimento realizado pelo SUS elevam as chances de ter consultado o médico nos últimos 12 meses do que

Tabela 1. Proporção de consulta ao médico nos últimos 12 meses, dos hipertensos e não hipertensos autorreferidos, por área no Brasil - Pesquisa Nacional por Amostra de Domicílios - 2008.

\begin{tabular}{lccccccc}
\hline $\begin{array}{c}\text { Situação do } \\
\text { domicílio }\end{array}$ & $\begin{array}{c}\text { Distribuição Proporção de } \\
\text { relativa (\%) }\end{array}$ & $\begin{array}{c}\text { OR * } \\
\text { consulta ao } \\
\text { médico (\%) }\end{array}$ & $\begin{array}{c}\text { Proporção de } \\
\text { consulta ao } \\
\text { médico (\%) }\end{array}$ & OR $^{*}$ & $\begin{array}{c}\text { Proporção de } \\
\text { consulta ao } \\
\text { médico (\%) }\end{array}$ & OR $^{*}$ \\
\hline Área urbana & 85,0 & 88,2 & $1,39(1,29-1,51)$ & 67,4 & $1,48(1,40-1,55)$ & 71,8 & $1,46(1,39-1,53)$ \\
Área rural & 15,0 & 84,3 & 1 & 58,4 & 1 & 63,6 & 1 \\
Total & 100 & 87,6 & & 66,1 & & 70,6 & \\
\hline
\end{tabular}

${ }^{*}$ IC $95 \%$. 
Tabela 2. Associações de condições demográficas e socioeconômicas com consulta ao médico nos últimos 12 meses - Pesquisa Nacional por Amostra de Domicílios - 2008.

\begin{tabular}{|c|c|c|c|c|}
\hline \multirow[b]{2}{*}{ Condições } & \multirow[b]{2}{*}{$\begin{array}{l}\text { Distribuição } \\
\text { relativa }(\%)\end{array}$} & \multirow[b]{2}{*}{$\begin{array}{l}\text { Proporção de } \\
\text { residentes em } \\
\text { área urbana }(\%)\end{array}$} & \multicolumn{2}{|c|}{$\begin{array}{l}\text { Associação com consulta ao } \\
\text { médico nos últimos } 12 \text { meses }\end{array}$} \\
\hline & & & $\begin{array}{l}\text { Proporção de } \\
\text { consulta }(\%)\end{array}$ & Odds ratio \\
\hline \multicolumn{5}{|l|}{ Região geográfica } \\
\hline Norte & 7,1 & 79,4 & 64,1 & 1 \\
\hline Nordeste & 26,5 & 74,2 & 66,9 & $1,13(1,05-1,21)$ \\
\hline Sudeste & 44,2 & 92,7 & 73,5 & $1,55(1,45-1,66)$ \\
\hline Sul & 15,0 & 83,2 & 72,2 & $1,45(1,35-1,57)$ \\
\hline Centro-oeste & 7,2 & 87,8 & 69,2 & $1,26(1,17-1,35)$ \\
\hline \multicolumn{5}{|l|}{ Sexo } \\
\hline Masculino & 47,5 & 83,5 & 58,8 & 1 \\
\hline Feminino & 52,5 & 86,4 & 81,2 & $3,03(2,97-3,10)$ \\
\hline \multicolumn{5}{|l|}{ Faixa etária } \\
\hline 20 a 35 anos & 39,8 & 85,4 & 64,5 & 1 \\
\hline 36 a 50 anos & 30,5 & 85,6 & 70,5 & $1,33(1,30-1,37)$ \\
\hline 51 a 64 anos & 18,2 & 84,4 & 76,6 & $1,79(1,74-1,84)$ \\
\hline 65 anos ou + & 11,5 & 83,5 & 82,3 & $2,36(2,27-2,45)$ \\
\hline \multicolumn{5}{|l|}{ Cor/raça } \\
\hline Branca & 50,2 & 87,9 & 72,7 & $1,23(1,20-1,26)$ \\
\hline Não branca & 49,8 & 82,2 & 68,4 & 1 \\
\hline \multicolumn{5}{|l|}{ Anos de estudo } \\
\hline Sem instrução ou menos de 1 ano & 12,3 & 67,5 & 69,0 & 1 \\
\hline 1 a 7 anos & 34,4 & 78,5 & 69,5 & $1,03(0,99-1,06)$ \\
\hline 8 a 14 anos & 44,5 & 92,6 & 70,0 & $1,05(1,01-1,09)$ \\
\hline 15 anos ou + & 8,8 & 96,9 & 80,0 & $1,70(1,61-1,79)$ \\
\hline \multicolumn{5}{|l|}{ Renda familiar per capita } \\
\hline $\begin{array}{l}\text { Sem rendimento ou até } 1 \text { salário } \\
\text { mínimo }\end{array}$ & 49,8 & 77,4 & 66,8 & 1 \\
\hline De 1 a 5 salários mínimos & 41,4 & 92,2 & 73,6 & $1,39(1,35-1,42)$ \\
\hline Mais de 5 salários mínimos & 5,2 & 97,4 & 82,5 & $2,34(2,21-2,48)$ \\
\hline Sem declaração & 3,6 & 91,1 & 71,2 & $1,23(1,15-1,32)$ \\
\hline
\end{tabular}

*Consultou vs. não consultou o médico nos últimos 12 meses

os que pagaram a consulta, chegando a 5 vezes mais entre os não hipertensos da área rural. As chances aumentam em mais de 3 vezes se o financiamento foi feito por plano de saúde, na área urbana, com relação aos que pagaram a consulta. Verifica-se que as mulheres apresentam mais chance de ter consultado o médico nos últimos 12 meses dos que os homens, em ambas as áreas, e esta chance aumenta sobretudo entre as mulheres não hipertensas (Tabela 5).

Apenas as regiões Nordeste, entre não hipertensos da área urbana, e Sudeste, entre os não hipertensos das duas áreas e entre os hipertensos da área urbana, apresentaram associações significativas com consulta ao médico nos últimos 12 meses, considerando a Região Norte como refe- rência. As pessoas que residem em domicílio cadastrado no PSF tem mais chance de ter consultado o médico nos últimos 12 meses. Em ambas as áreas, pessoas com autopercepção de saúde muito boa/boa ou regular apresentaram menores chances de consultar ao médico, com relação aos que referiram a autopercepção de saúde como ruim ou muito ruim, em ambos os estratos de HAS. Ser portador de alguma morbidade eleva a chance de consulta ao médico, em ambas as áreas, com uma intensidade ligeiramente maior entre os não hipertensos. Pessoas que possuem alguma limitação têm mais chance de terem consultado o médico. Em todos os estratos, os que possuem plano de saúde apresentaram chances maiores de consulta ao médico nos últimos 12 meses do que 
Tabela 3. Associações de condições de saúde com consulta ao médico nos últimos 12 meses - Pesquisa Nacional por Amostra de Domicílios - 2008.

\begin{tabular}{|c|c|c|c|c|}
\hline \multirow[b]{2}{*}{ Condições } & \multirow[b]{2}{*}{$\begin{array}{c}\text { Distribuição } \\
\text { relativa (\%) }\end{array}$} & \multirow[b]{2}{*}{$\begin{array}{l}\text { Proporção de } \\
\text { residentes em } \\
\text { área urbana }(\%)\end{array}$} & \multicolumn{2}{|c|}{$\begin{array}{l}\text { Associação com consulta ao } \\
\text { médico nos últimos } 12 \text { meses }\end{array}$} \\
\hline & & & $\begin{array}{l}\text { Proporção de } \\
\text { consulta }(\%)\end{array}$ & Odds ratio* \\
\hline \multicolumn{5}{|l|}{ O domicílio cadastrado no PSF } \\
\hline Sim & 48,7 & 79,2 & 70,2 & $0,96(0,93-0,99)$ \\
\hline Não & 51,3 & 90,6 & 71,0 & 1 \\
\hline \multicolumn{5}{|l|}{ Hipertensão autorreferida } \\
\hline Sim & 20,9 & 85,6 & 87,6 & $3,63(3,52-3,75)$ \\
\hline Não & 79,1 & 84,9 & 66,1 & 1 \\
\hline \multicolumn{5}{|l|}{ Morbidade autorreferida } \\
\hline $\begin{array}{l}\text { Diabet./doen. coração/insuf. renal } \\
\text { crônica }\end{array}$ & 4,8 & 86,7 & 86,7 & $3,12(2,96-3,29)$ \\
\hline Outra doença ${ }^{* *}$ & 29,8 & 85,3 & 84,4 & $2,80(2,73-2,87)$ \\
\hline Sem morbidade ${ }^{* * *}$ & 65,4 & 84,8 & 63,1 & 1 \\
\hline \multicolumn{5}{|l|}{ Autopercepção de saúde } \\
\hline Muito bom/Bom & 70,4 & 86,9 & 65,3 & $0,22(0,21-0,24)$ \\
\hline Regular & 24,3 & 80,8 & 81,8 & $0,53(0,50-0,57)$ \\
\hline Muito ruim/Ruim & 5,3 & 80,2 & 89,4 & 1 \\
\hline \multicolumn{5}{|l|}{ Mobilidade física } \\
\hline Sem limitação & 67,4 & 86,0 & 64,4 & 1 \\
\hline Pouco limitada & 26,5 & 82,9 & 83,1 & $2,46(2,39-2,54)$ \\
\hline Limitada & 6,1 & 83,3 & 84,8 & $2,68(2,55-2,81)$ \\
\hline \multicolumn{5}{|l|}{ Medicamentos de uso contínuo } \\
\hline Sim & 28,6 & 87,1 & 91,0 & $3,96(3,86-4,06)$ \\
\hline Não & 71,4 & 84,2 & 62,4 & 1 \\
\hline \multicolumn{5}{|l|}{ Tem direito a plano de saúde } \\
\hline Sim & 28,4 & 96,1 & 82,1 & $2,37(2,30-2,44)$ \\
\hline Não & 71,6 & 80,7 & 66,0 & 1 \\
\hline \multicolumn{5}{|l|}{ Financiamento a atendimento de saúde } \\
\hline Pagou & 3,1 & 89,7 & 91,3 & 1 \\
\hline Plano de saúde & 3,5 & 97,8 & 98,4 & $6,03(4,86-7,47)$ \\
\hline SUS & 8,2 & 83,1 & 97,2 & $3,34(2,91-3,83)$ \\
\hline Não procurou ou não foi atendido & 84,5 & 84,5 & 65,9 & $0,18(0,17-0,20)$ \\
\hline Sem declaração & 0,7 & 87,4 & 89,4 & $0,80(0,67-0,96)$ \\
\hline
\end{tabular}

* Consultou vs. não consultou o médico nos últimos 12 meses. ${ }^{* *}$ Doença de coluna ou costas, artrite ou reumatismo, câncer, bronquite ou asma, depressão, tuberculose, tendinite ou tenossinovite ou cirrose. ${ }^{\star * *}$ A HAS não foi considerada.

os que não possuem, sendo um pouco maior entre os não hipertensos da área urbana (Tabela 5).

A análise múltipla modificou as relações de todas as variáveis. A magnitude da associação da região geográfica com HAS apresentada na Tabela 4, considerando a Região Norte como referência, reduz em todos os estratos no modelo multivariado (Tabela 5).

A maior chance de consulta ao médico por mulheres com relação aos homens observada na Tabela 4 se atenua em todos os estratos, com a maior diferença observada entre os hipertensos da área rural, que passou de $\mathrm{OR}_{\text {bruto }}=1,94$ para $\mathrm{OR}_{\text {ajustado }}=1,59$, quando controlada pelas outras variáveis. As variáveis renda familiar per capita, morbidade autorreferida, autopercepção de saúde e mobilidade física também apresentam uma redução na magnitude da associação na análise múltipla (Tabela 5).

A variável cor/raça apenas não perde a associação na análise multivariada entre os não hipertensos da área urbana, entretanto esta associação é bastante fraca $(\mathrm{OR}=0,95)$, considerando a categoria não branca como referência (Tabela 5). As variáveis medicamentos de uso contínuo e plano de saúde apresentaram no modelo multivariado apenas uma pequena redução da associação, com relação à análise univariada (Tabela 4). 
Tabela 4. Modelos logísticos univariados para consulta ao médico nos últimos 12 meses dos hipertensos e não hipertensos autorreferidos, segundo situação do domicílio - Pesquisa Nacional por Amostra de Domicílios - 2008.

\begin{tabular}{|c|c|c|c|c|}
\hline \multirow[b]{3}{*}{ Características } & \multicolumn{4}{|c|}{ Modelos logísticos univariados } \\
\hline & \multicolumn{2}{|c|}{ Hipertensos autorreferidos } & \multicolumn{2}{|c|}{ Não hipertensos autorreferidos } \\
\hline & $\begin{array}{c}\text { Urbana } \\
\text { OR }^{\star}(\text { IC 95\%) }\end{array}$ & $\begin{array}{c}\text { Rural } \\
\text { OR }^{\star}(\text { IC 95\%) }\end{array}$ & $\begin{array}{c}\text { Urbana } \\
\text { OR }^{\star}(\text { IC } 95 \%)\end{array}$ & $\begin{array}{c}\text { Rural } \\
\text { OR }^{\star}(\text { IC 95\%) }\end{array}$ \\
\hline \multicolumn{5}{|l|}{ Região geográfica } \\
\hline Norte & 1 & 1 & 1 & 1 \\
\hline Nordeste & $1,11(0,98-1,25)$ & $1,17(0,95-1,45)$ & $1,12(1,04-1,20)$ & $1,07(0,88-1,30)$ \\
\hline Sudeste & $1,43(1,27-1,62)$ & $1,27(1,00-1,61)$ & $1,40(1,31-1,50)$ & $1,40(1,14-1,72)$ \\
\hline Sul & $1,26(1,09-1,47)$ & $1,47(1,14-1,89)$ & $1,35(1,25-1,47)$ & $1,42(1,15-1,75)$ \\
\hline Centro-oeste & $1,01(0,88-1,16)$ & $1,09(0,79-1,51)$ & $1,21(1,12-1,30)$ & $1,16(0,93-1,46)$ \\
\hline \multicolumn{5}{|c|}{ 然 } \\
\hline Masculino & 1 & 1 & 1 & 1 \\
\hline Feminino & $2,13(2,00-2,27)$ & $1,94(1,72-2,20)$ & $3,05(2,97-3,12)$ & $3,08(2,90-3,26)$ \\
\hline \multicolumn{5}{|l|}{ Faixa etária } \\
\hline 20 a 35 anos & 1 & 1 & 1 & 1 \\
\hline 36 a 50 anos & $1,33(1,19-1,48)$ & $1,00(0,82-1,22)$ & $1,20(1,17-1,24)$ & $1,01(0,95-1,06)$ \\
\hline 51 a 64 anos & $1,56(1,41-1,73)$ & $1,26(1,03-1,54)$ & $1,34(1,29-1,39)$ & $1,05(0,99-1,12)$ \\
\hline 65 anos ou + & $1,94(1,74-2,18)$ & $1,31(1,04-1,65)$ & $1,67(1,58-1,75)$ & $1,25(1,14-1,37)$ \\
\hline \multicolumn{5}{|c|}{ 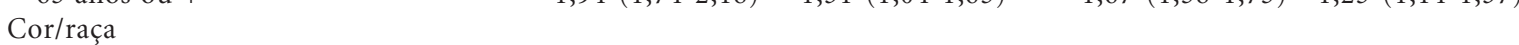 } \\
\hline Branca & $1,13(1,06-1,21)$ & $1,20(1,03-1,39)$ & $1,22(1,19-1,25)$ & $1,21(1,12-1,30)$ \\
\hline Não branca & 1 & 1 & 1 & 1 \\
\hline \multicolumn{5}{|l|}{ Anos de estudo } \\
\hline Sem instrução ou menos de 1 ano & 1 & 1 & 1 & 1 \\
\hline 1 a 7 anos & $1,02(0,94-1,11)$ & $1,13(0,97-1,30)$ & $1,13(1,09-1,19)$ & $1,18(1,10-1,26)$ \\
\hline 8 a 14 anos & $0,95(0,87-1,05)$ & $1,23(0,97-1,56)$ & $1,32(1,26-1,38)$ & $1,47(1,36-1,59)$ \\
\hline 15 anos ou + & $1,08(0,94-1,24)$ & $1,27(0,66-2,44)$ & $2,31(2,17-2,46)$ & $2,30(1,88-2,82)$ \\
\hline \multicolumn{5}{|l|}{ Renda familiar per capita } \\
\hline $\begin{array}{l}\text { Sem rendimento ou até } 1 \text { salário } \\
\text { mínimo }\end{array}$ & 1 & 1 & 1 & 1 \\
\hline De 1 a 5 salários mínimos & $1,21(1,13-1,30)$ & $1,13(0,96-1,33)$ & $1,35(1,31-1,39)$ & $1,33(1,23-1,44)$ \\
\hline Mais de 5 salários mínimos & $1,52(1,30-1,77)$ & $1,39(0,72-2,69)$ & $2,19(2,06-2,32)$ & $1,76(1,30-2,39)$ \\
\hline Sem declaração & $1,10(0,92-1,30)$ & $0,85(0,55-1,31)$ & $1,21(1,12-1,31)$ & $1,08(0,88-1,32)$ \\
\hline \multicolumn{5}{|l|}{ O domicílio cadastrado no PSF } \\
\hline Sim & $1,08(1,00-1,16)$ & $1,20(1,03-1,40)$ & $0,95(0,92-0,98)$ & $1,14(1,04-1,25)$ \\
\hline Não & 1 & 1 & 1 & 1 \\
\hline \multicolumn{5}{|l|}{ Autopercepção de saúde } \\
\hline Muito bom/Bom & $0,37(0,33-0,42)$ & $0,37(0,28-0,48)$ & $0,29(0,26-0,31)$ & $0,25(0,22-0,29)$ \\
\hline Regular & $0,62(0,55-0,70)$ & $0,56(0,43-0,73)$ & $0,59(0,54-0,64)$ & $0,55(0,48-0,64)$ \\
\hline Muito ruim/Ruim & 1 & 1 & 1 & 1 \\
\hline \multicolumn{5}{|l|}{ Mobilidade física } \\
\hline Sem limitação & 1 & 1 & 1 & 1 \\
\hline Pouco limitada & $2,02(1,88-2,17)$ & $1,85(1,60-2,15)$ & $2,09(2,01-2,17)$ & $2,26(2,10-2,43)$ \\
\hline Limitada & $2,45(2,17-2,77)$ & $2,56(2,01-3,25)$ & $2,14(2,01-2,29)$ & $2,35(2,06-2,69)$ \\
\hline \multicolumn{5}{|l|}{ Morbidade autorreferida } \\
\hline $\begin{array}{l}\text { Diabet./doen. coração/insuf. renal } \\
\text { crônica }\end{array}$ & $2,15(1,92-2,40)$ & $2,00(1,56-2,57)$ & $2,54(2,35-2,75)$ & $3,42(2,82-4,13)$ \\
\hline Outra doença ${ }^{* *}$ & $2,33(2,18-2,49)$ & $1,90(1,65-2,18)$ & $2,44(2,37-2,52)$ & $2,73(2,55-2,93)$ \\
\hline Sem morbidade $* * *$ & 1 & 1 & 1 & 1 \\
\hline \multicolumn{5}{|l|}{ Medicamentos de uso contínuo } \\
\hline Sim & $4,23(3,94-4,54)$ & $3,88(3,33-4,51)$ & $3,49(3,35-3,62)$ & $7,08(6,23-8,05)$ \\
\hline Não & 1 & 1 & 1 & 1 \\
\hline \multicolumn{5}{|l|}{ Tem direito a plano de saúde } \\
\hline Sim & $1,88(1,74-2,03)$ & $1,67(1,29-2,16)$ & $2,27(2,20-2,34)$ & $2,15(1,89-2,44)$ \\
\hline Não & 1 & 1 & 1 & 1 \\
\hline \multicolumn{5}{|l|}{ Financiamento do último atendimento } \\
\hline Pagou & 1 & 1 & 1 & 1 \\
\hline Plano de saúde & $5,22(2,75-9,91)$ & $1,81(0,32-10,14)$ & $1,47(1,34-1,61)$ & $3,26(1,28-8,28)$ \\
\hline SUS & $2,52(1,71-3,71)$ & $3,09(1,34-7,11)$ & $1,35(1,23-1,48)$ & $4,39(3,05-6,32)$ \\
\hline Não procurou ou não foi atendido & $0,16(0,12-0,22)$ & $0,16(0,08-0,33)$ & $0,30(0,28-0,32)$ & $0,22(0,17-0,28)$ \\
\hline Sem declaração & $0,58(0,34-0,99)$ & $0,46(0,18-1,15)$ & $0,89(0,76-1,06)$ & $1,04(0,63-1,72)$ \\
\hline
\end{tabular}

* OR ajustado (consultou vs. não consultou o médico nos últimos 12 meses). " Doença de coluna ou costas, artrite ou reumatismo, câncer, bronquite ou asma, depressão, tuberculose, tendinite ou tenossinovite ou cirrose. ${ }^{* *}$ A HAS não foi considerada 
Tabela 5. Modelos logísticos multivariados para consulta ao médico nos últimos 12 meses dos hipertensos e não hipertensos autorreferidos, segundo situação do domicílio - Pesquisa Nacional por Amostra de Domicílios - 2008.

\begin{tabular}{|c|c|c|c|c|}
\hline \multirow[b]{3}{*}{ Características } & \multicolumn{4}{|c|}{ Modelos logísticos multivariados } \\
\hline & \multicolumn{2}{|c|}{ Hipertensos autorreferidos } & \multicolumn{2}{|c|}{ Não hipertensos autorreferidos } \\
\hline & $\begin{array}{c}\text { Urbana } \\
\mathrm{OR}_{\mathrm{aj}}^{*}(\mathrm{IC} 95 \%)\end{array}$ & $\underset{\mathrm{OR}_{\mathrm{aj}}^{*}}{\stackrel{\text { Rural }}{(\mathrm{IC} 95 \%)}}$ & $\begin{array}{c}\text { Urbana } \\
\mathrm{OR}_{\mathrm{aj}}^{*}(\mathrm{IC} 95 \%)\end{array}$ & $\begin{array}{c}\text { Rural } \\
\mathrm{OR}_{\text {aj }}^{*}(\mathrm{IC} 95 \%)\end{array}$ \\
\hline \multicolumn{5}{|l|}{ Região geográfica } \\
\hline Norte & 1 & 1 & 1 & 1 \\
\hline Nordeste & $1,03(0,91-1,18)$ & $1,09(0,85-1,38)$ & $1,09(1,01-1,16)$ & $1,06(0,89-1,24)$ \\
\hline Sudeste & $1,15(1,01-1,30)$ & $0,95(0,73-1,24)$ & $1,15(1,07-1,22)$ & $1,23(1,03-1,46)$ \\
\hline Sul & $0,94(0,81-1,11)$ & $1,00(0,74-1,36)$ & $1,06(0,98-1,15)$ & $1,14(0,96-1,36)$ \\
\hline Centro-oeste & $0,88(0,76-1,02)$ & $0,92(0,67-1,26)$ & $1,05(0,98-1,13)$ & $1,06(0,88-1,27)$ \\
\hline \multicolumn{5}{|c|}{ ו } \\
\hline Masculino & 1 & 1 & 1 & 1 \\
\hline Feminino & $1,83(1,71-1,95)$ & $1,59(1,40-1,80)$ & $2,81(2,73-2,89)$ & $2,78(2,62-2,96)$ \\
\hline \multicolumn{5}{|l|}{ Faixa etária } \\
\hline 20 a 35 anos & 1 & 1 & 1 & 1 \\
\hline 36 a 50 anos & $0,87(0,77-0,98)$ & $0,73(0,59-0,91)$ & $1,02(0,99-1,05)$ & $0,84(0,78-0,90)$ \\
\hline 51 a 64 anos & $0,72(0,63-0,81)$ & $0,72(0,58-0,91)$ & $0,93(0,88-0,97)$ & $0,76(0,70-0,82)$ \\
\hline 65 anos ou + & $0,71(0,62-0,82)$ & $0,66(0,50-0,86)$ & $0,89(0,83-0,95)$ & $0,73(0,64-0,83)$ \\
\hline \multicolumn{5}{|l|}{ Cor/raça } \\
\hline Branca & $0,97(0,90-1,05)$ & $1,07(0,91-1,26)$ & $0,95(0,92-0,97)$ & $0,99(0,92-1,06)$ \\
\hline Não branca & 1 & 1 & 1 & 1 \\
\hline \multicolumn{5}{|l|}{ Anos de estudo } \\
\hline Sem instrução ou menos de 1 ano & 1 & 1 & 1 & 1 \\
\hline 1 a 7 anos & $1,10(1,00-1,21)$ & $1,19(1,02-1,39)$ & $1,32(1,26-1,39)$ & $1,19(1,10-1,29)$ \\
\hline 8 a 14 anos & $1,15(1,03-1,29)$ & $1,47(1,13-1,90)$ & $1,50(1,42-1,59)$ & $1,43(1,30-1,57)$ \\
\hline 15 anos ou + & $1,11(0,93-1,32)$ & $1,33(0,77-2,27)$ & $1,82(1,69-1,96)$ & $1,85(1,51-2,27)$ \\
\hline \multicolumn{5}{|l|}{ Renda familiar per capita } \\
\hline $\begin{array}{l}\text { Sem rendimento ou até } 1 \text { salário } \\
\text { mínimo }\end{array}$ & 1 & 1 & 1 & 1 \\
\hline De 1 a 5 salários mínimos & $1,06(0,97-1,14)$ & $0,98(0,83-1,16)$ & $1,12(1,08-1,15)$ & $1,22(1,12-1,32)$ \\
\hline Mais de 5 salários mínimos & $1,13(0,94-1,36)$ & $1,30(0,72-2,36)$ & $1,37(1,27-1,47)$ & $1,14(0,84-1,55)$ \\
\hline Sem declaração & $0,91(0,76-1,10)$ & $0,90(0,60-1,35)$ & $0,95(0,87-1,04)$ & $1,04(0,85-1,27)$ \\
\hline \multicolumn{5}{|l|}{ O domicílio cadastrado no PSF } \\
\hline Sim & $1,16(1,07-1,25)$ & $1,16(0,99-1,36)$ & $1,12(1,08-1,16)$ & $1,21(1,10-1,33)$ \\
\hline Não & 1 & 1 & 1 & 1 \\
\hline \multicolumn{5}{|l|}{ Autopercepção de saúde } \\
\hline Muito bom/Bom & $0,65(0,57-0,75)$ & $0,66(0,50-0,88)$ & $0,55(0,49-0,60)$ & $0,55(0,47-0,65)$ \\
\hline Regular & $0,83(0,72-0,94)$ & $0,79(0,61-1,02)$ & $0,78(0,71-0,87)$ & $0,85(0,72-1,00)$ \\
\hline Muito ruim/Ruim & 1 & 1 & 1 & 1 \\
\hline \multicolumn{5}{|l|}{ Mobilidade física } \\
\hline Sem limitação & 1 & 1 & 1 & 1 \\
\hline Pouco limitada & $1,28(1,18-1,40)$ & $1,30(1,10-1,53)$ & $1,34(1,28-1,40)$ & $1,42(1,31-1,53)$ \\
\hline Limitada & $1,32(1,15-1,52)$ & $1,49(1,17-1,89)$ & $1,24(1,15-1,35)$ & $1,24(1,07-1,44)$ \\
\hline \multicolumn{5}{|l|}{ Morbidade autorreferida } \\
\hline $\begin{array}{l}\text { Diabet./doen. coração/insuf. renal } \\
\text { crônica }\end{array}$ & $1,49(1,32-1,68)$ & $1,35(1,04-1,74)$ & $1,62(1,47-1,79)$ & $1,85(1,51-2,27)$ \\
\hline Outra doença ${ }^{* *}$ & $1,41(1,30-1,52)$ & $1,32(1,13-1,54)$ & $1,64(1,58-1,70)$ & $1,73(1,61-1,86)$ \\
\hline Sem morbidade $* * *$ & 1 & 1 & 1 & 1 \\
\hline \multicolumn{5}{|l|}{ Medicamentos de uso contínuo } \\
\hline Sim & $3,13(2,88-3,41)$ & $3,12(2,69-3,62)$ & $2,88(2,72-3,06)$ & $3,52(3,09-4,03)$ \\
\hline Não & 1 & 1 & 1 & 1 \\
\hline \multicolumn{5}{|l|}{ Tem direito a plano de saúde } \\
\hline Sim & $1,79(1,63-1,97)$ & $1,49(1,13-1,96)$ & $2,15(2,07-2,23)$ & $1,76(1,53-2,03)$ \\
\hline Não & 1 & 1 & 1 & 1 \\
\hline \multicolumn{5}{|l|}{ Financiamento do último atendimento } \\
\hline Pagou & 1 & 1 & 1 & 1 \\
\hline Plano de saúde & $3,50(1,84(6,68)$ & $1,36(0,26-7,03)$ & $3,65(2,87-4,64)$ & $2,05(1,00-4,23)$ \\
\hline SUS & $2,69(1,84-3,95)$ & $3,23(1,64-6,39)$ & $3,64(3,08-4,30)$ & $5,23(3,65-7,50)$ \\
\hline Não procurou ou não foi atendido & $0,22(0,16-0,29)$ & $0,23(0,14-0,39)$ & $0,29(0,26-0,33)$ & $0,35(0,27-0,44)$ \\
\hline Sem declaração & $0,75(0,44-1,28)$ & $0,53(0,24-1,16)$ & $1,09(0,87-1,35)$ & $1,25(0,74-2,10)$ \\
\hline
\end{tabular}

" OR ajustado (consultou vs. não consultou o médico nos últimos 12 meses). ${ }^{* *}$ Doença de coluna ou costas, artrite ou reumatismo, câncer, bronquite ou asma, depressão, tuberculose, tendinite ou tenossinovite ou cirrose. ${ }^{* * *}$ A HAS não foi considerada 
A associação da variável financiamento do último atendimento aumentou, após a análise múltipla apenas na categoria plano de saúde entre os não hipertensos da área urbana, com relação aos que pagaram consulta. Entretanto, na categoria SUS, há um aumento na magnitude da associação em todas as categorias, em comparação com a análise univariada. Nesta categoria, embora a associação seja forte, há um crescimento nos estratos da seguinte maneira: os não hipertensos possuem uma associação maior do que os hipertensos e o rural é sempre maior do que o urbano.

A variável anos de estudo, em geral, aumentou a associação, mas apresentou uma redução entre os não hipertensos com 15 anos de estudo ou mais, em ambas as áreas, e não hipertensos com 8 a 14 anos de estudo da área rural, na análise multivariada, considerando as pessoas sem instrução ou com a te 1 ano de estudo como referência (Tabela 5).

O Gráfico 1 ilustra a distribuição das proporções estimadas pelo modelo logístico, entre os que referiram e não referiram HAS, de acordo com a situação do domicílio urbana ou rural. Observase que entre os hipertensos da área urbana, $3 / 4$ deles têm proporção estimada maior do que $84 \%$ de ter consultado o médico nos últimos 12 meses. Entre os hipertensos da área rural esta proporção estimada é de 79\%. Verificam-se ainda casos extremos (outliers) entre os hipertensos. Valores inferiores a $66 \%$ podem ser considerados atípicos para hipertensos residentes em área urbana. Em área rural, os valores atípicos são os inferiores a $60 \%$. Entre os não hipertensos, observa-se que os residentes em área urbana têm maior tendência a ter consultado o médico do que os residentes na área rural e esta proporção é menor quando comparada aos hipertensos. Verifica-se também que entre os não hipertensos, as proporções estimadas de consulta médica apresentam mais variabilidade na distribuição das proporções que os hipertensos em ambas as áreas.

\section{Discussão}

O presente estudo mostrou que a proporção de consultas médicas nos últimos 12 meses foi de $70,6 \%$, sendo $71,8 \%$ na área urbana e 63,6\% na área rural. Proporções parecidas - em torno de $60 \%$ e $70 \%$ da população, em países como Estados Unidos, Noruega, Irlanda e Brasil - foram encontradas em revisão sistemática sobre utilização de serviços de saúde, realizada por Mendonza-Sassi e Béria ${ }^{19}$. Com relação ao resultado

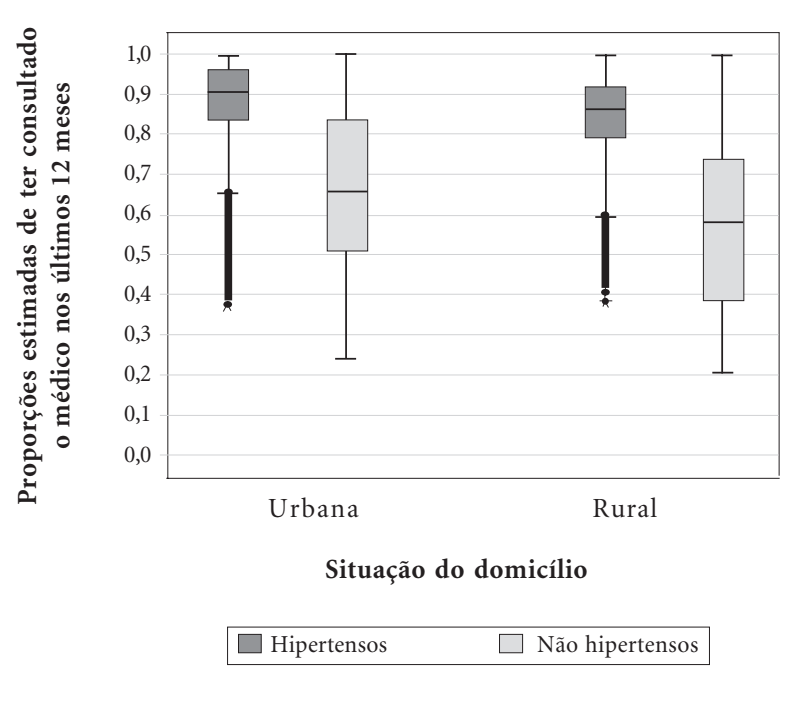

Gráfico 1. Box-plot entre a probabilidade de ter consultado o médico nos últimos 12 meses, entre os que referiram e não referiram HAS, por situação do domicílio.

da área rural, o acesso aos serviços de saúde tende a ser menor, seja pelas distâncias a serem percorridas, seja por dificuldade de transporte ou baixa renda ${ }^{7}$.

Entre os que referiram HAS, observou-se que a utilização de consulta médica nos últimos 12 meses é maior do que entre os que não referiram. Este resultado era esperado, tendo em vista que um dos determinantes para a utilização dos serviços é a necessidade de saúde ${ }^{6}$. Esta maior utilização foi encontrada em diversos estudos que consideraram, além da HAS, outras doenças crônicas $^{8,20}$. O fato que indivíduos que utilizam medicamentos de uso contínuo tenham apresentado elevada associação com consulta ao médico em todos os estratos pode ser explicado, parcialmente, pelo motivo de estes serem também portadores de doenças crônicas, necessitando, portanto, de controle médico.

Embora tenha sido verificado que a utilização de consulta médica é maior entre os HAS autorreferidos e entre residentes em área urbana, nem todas as variáveis se comportaram exatamente da mesma forma nestes dois grupos. Mesmo após a perda de magnitude de associação na análise múltipla, verificou-se que as mulheres têm mais chance de ter consultado o médico nos últimos 12 meses do que os homens, e esta relação é maior entre as que não referiram HAS. É de amplo conhecimento que as mulheres utilizam mais os serviços de saúde do que os 
homens, o que pode ser devido à maior preocupação com a saúde ou às necessidades relacionadas à reprodução. Além disso, as mulheres ainda tendem a referir mais problemas de saúde do que os homens ${ }^{3,21-24}$.

Os níveis mais elevados de faixa etária mostraram menor chance de ter consultado o médico nos últimos 12 meses do que dos indivíduos de 20 a 35 anos, após o controle para todas as variáveis. Diversos trabalhos encontraram que a consulta ao médico aumenta conforme a idade, entretanto esses modelos não foram controlados para todas as variáveis consideradas neste estudo. Costa et al. ${ }^{25}$, considerando as variáveis classe econômica, renda familiar, escolaridade, idade, cor da pele, estado civil, tabagismo, consumo de álcool, índice de massa corporal (IMC), diabetes autorreferida, HAS, distúrbios psiquiátricos menores, bronquite crônica e internação hospitalar, concluíram que os idosos consultam mais ao médico. Mendoza-Sassi et al. ${ }^{26}$ chegaram a esta mesma conclusão ao ajustar um modelo multivariado para as seguintes variáveis: sexo, faixa etária, renda per capita, escolaridade, eventos estressantes, serviço de saúde regular, autopercepção de saúde e dias inativos. Contudo, os resultados deste estudo sugerem que, provavelmente, não é a idade que determina a consulta ao médico e, sim, a condição de saúde, mais particularmente a prevalência de doenças crônicas ${ }^{10,27}$. Foram testadas interações entre a variável faixa etária e as variáveis de saúde: autopercepção de saúde, mobilidade física, morbidade autorreferida, medicamentos de uso contínuo e tem direito a plano de saúde. Entretanto, estas não apresentaram efeito estatisticamente significante.

Observou-se que, quanto mais anos de estudo, maiores chances de ter consultado o médico nos últimos 12 meses, entre os que não referiram HAS, residentes em ambas as áreas. No geral, a magnitude destas associações aumentou na análise multivariada em comparação com a univariada. Já entre os não hipertensos com 15 anos de estudo ou mais nas duas áreas e entre os de 8 a 14 anos na área rural, foi observada uma redução da magnitude da associação após a análise multivariada, com relação aos indivíduos sem instrução ou com até 1 ano de estudo. Maior escolaridade implica em mais informação e mais recursos de comunicação, que favorecem o acesso aos serviços de saúde ${ }^{7}$. Capilheira e Santos ${ }^{28}$ apontam que resultados de estudos que consideram a variável anos de estudo têm sido controversos, mostrando que ora a escolaridade favorece a consulta ao médico, ora não influencia, como podem também ser observados em Mendoza-Sassi et al. ${ }^{19}$ e Costa e Facchini ${ }^{29}$.

Verificou-se maior utilização de consultas médicas por indivíduos que se declaram com a saúde ruim/muito ruim, tanto entre hipertensos como entre não hipertensos, nas duas áreas. Esta associação, que era bem forte na análise bruta, manteve o sentido, mas diminuiu a magnitude da associação após o controle por todas as variáveis. Outros estudos que utilizaram esta variável também sinalizam que indivíduos com autopercepção do estado de saúde ruim ou muito ruim têm mais chance de consultar o médico ${ }^{26}$ do que aqueles que se declaram com saúde boa/ muito boa ou regular.

Existe uma associação entre plano de saúde e consulta ao médico nos últimos 12 meses. A população que possuía financiamento no último atendimento tem mais chance de consultar ao médico do que aqueles que pagaram pela consulta. Em estudo realizado por Pinheiro et al. ${ }^{3}$, utilizando dados da PNAD realizada em 1998 sugeriu que quem tem plano faz uso prioritário deste, independentemente de viver em área urbana ou rural. Os resultados encontrados sugerem o fenômeno conhecido como risco moral (moral hazard), que é o incremento da exposição aos riscos e o uso excessivo de serviços cobertos pelos seguros por parte dos segurados quando existe um financiador, como o governo ou operadoras privadas ${ }^{30}$.

Alguns estudos elucidam associação entre consulta ao médico com renda e com cor/ raça, sugerindo que a cor branca e maior renda estão associadas ao maior utilização de consultas médicas ${ }^{25,26,28,31,32}$. Após a análise múltipla, este estudo apontou associações significativas nesta mesma direção apenas para a variável renda familiar per capita, em que foi observado um gradiente entre os não hipertensos na área urbana que recebem de 1 a 5 e mais de 5 salários mínimos, com relação aos que não possuem rendimento ou recebem até 1 salário mínimo.

Indivíduos com alguma limitação na mobilidade física, em todos os estratos, apresentaram maior associação com consulta ao médico, que pode ser devido, provavelmente, à própria limitação, que impõe mais necessidade de saúde do que pessoas que não apresentam limitação.

Os dados da PNAD vêm sendo cada vez mais utilizado em estudos na área da saúde, ainda que poucos explorem a situação urbana e rural. A maioria dos trabalhos que utilizam a PNAD tem como objetivo apenas descrever os dados, sendo necessário, para isso, apenas a incorporação dos 
pesos amostrais ${ }^{10,33}$. Diferentemente destes, o presente estudo, que utiliza modelagem, incorporou o esquema amostral complexo da pesquisa, pois estes tipos de dados não podem ser tratados como se fossem observações independentes e identicamente distribuídas, como fazem os procedimentos usuais de análise disponíveis nos pacotes estatísticos ${ }^{15,18}$. Utilizou também os pesos relativos, visando conservar o tamanho amostral e consequente manutenção da precisão dos estimadores.

Representativa da população brasileira, a PNAD permite fazer inferências para todo o território nacional. Outra vantagem da utilização desta pesquisa é que o uso de dados secundários economiza tempo e custo, em virtude de já terem sido coletados ${ }^{34}$.

A PNAD utiliza a morbidade autorreferida para mensurar doenças crônicas na população, inclusive a HAS. Esta medida é comumente utilizada em inquéritos populacionais, em virtude de ser uma medida rápida e de baixo custo. Entretanto, como não utiliza nenhum meio para verificação ou conferência desta informação, é sujeita a viés de informação ${ }^{35}$. No caso da hipertensão, em que o paciente só reconhece o problema após o diagnóstico médico, os inquéritos que utilizam de morbidade autorreferida tendem a subestimar sua prevalência ${ }^{10}$. Wu et al. ${ }^{36}$, em estudo realizado em Taiwan, estimaram que a prevalência tende a ser subestimada em $4 \%$. Contudo, vários estudos sugerem validade dos resultados que utilizam medidas de indivíduos que se declararam hipertensos para grandes populações ${ }^{11-13}$.

Este estudo apresenta limitações com relação à classificação da população em residentes em área urbana e em área rural. O ponto crítico que separa o que é urbano do que é rural é arbitrário. Cada país possui uma forma de classificação de acordo com as suas regras, e cada definição tem sido de- senvolvida com propostas administrativas, políticas ou geográficas ${ }^{37,38}$. No Brasil, a classificação das populações urbana e rural é administrativa e tem por base a legislação vigente por ocasião da realização do Censo Demográfico 2000. Como situação urbana, consideram-se as áreas correspondentes às cidades, às vilas ou às áreas urbanas isoladas. A situação rural abrange toda a área situada fora desses limites. Esta classificação se baseia na distância até a sede municipal ou distrital e, em decorrência disto, considera como urbanos em torno de $90 \%$ dos municípios do Brasil ${ }^{14}$. Neste sentido, é provável que haja uma superestimativa de municípios considerados urbanos.

Outra limitação está relacionada a potenciais variáveis importantes que não foram incorporadas na análise, como o preço do tratamento e o tempo gasto para chegar ao local do atendimento $^{39}$, em virtude de não estarem disponíveis na PNAD. Por outro lado, a técnica de análise de sensibilidade poderia ser utilizada para avaliar o impacto no resultado do estudo, tanto em termos de variáveis não mensuradas, quanto de diferentes definições de ambiente urbano e rural ${ }^{40}$.

Observa-se, neste estudo, uma associação fraca de diversas variáveis com o consulta ao médico (menor do que 2). Entretanto, estes resultados não significam que não existe relevância do problema de saúde sob o ponto de vista de políticas públicas ${ }^{41}$.

O presente artigo identificou que o principal determinante para consulta ao médico foram as necessidades de saúde. Os hipertensos consultaram mais ao médico do que os não hipertensos, independentemente da idade. Residentes em área urbana também têm mais chance de ter consultado o médico e estas diferenças entre as áreas urbanas e rurais sugerem que políticas de acesso devem ser implantadas, com o objetivo de reduzir as iniquidades em saúde. 


\section{Colaboradores}

JPL Moreira, JR Moraes e RR Luiz participaram igualmente de todas as etapas de elaboração do artigo.

\section{Agradecimentos}

Este projeto foi parcialmente financiado com recurso da Faperj.

\section{Referências}

1. Donabedian A. Explorations in quality assessment and monitoring: the definition of quality and approaches to its assessment. Ann Arbor, MI: Health Administration Press; 1980.

2. Oliveira EXG, Carvalho MS, Travassos C. Territórios do Sistema Único de Saúde: mapeamento das redes de atenção hospitalar. Cad Saude Publica 2004; 20(2):386-402.

3. Pinheiro RS, Viacava F, Travassos C, Brito AS. Gênero, morbidade, acesso e utilização de serviços de saúde no Brasil. Cien Saude Colet 2002; 7(4):687-707.

4. Ojanuga DN; Gilbert C. Women's access to health care in developing countries. Social Science and Medicine 1992; 35(4):613-617.

5. Puentes-Markides C. Women and access to health care. Social Science and Medicine 1992; 35(4):619-626.

6. Hulka BS, Wheat JR. Patterns of utilization: the patient perspective. Medical Care 1985, 23(5):438-460.

7. Travassos C, Viacava F. Acesso e uso de serviços de saúde em idosos residentes em áreas rurais, Brasil, 1998 e 2003. Cad Saude Publica 2007; 23(10):24902502.

8. Almeida MF, Barata RB, Montero CV, Silva ZP. Prevalência de doenças crônicas auto-referidas e utilização de serviços de saúde, PNAD/1998, Brasil. Cien Saude Colet 2005; 7(4):743-756.

9. Organização Pan-americana de Saúde (OPAS)/Organização Mundial de Saúde (OMS). Prevenção e Controle de Doenças e Desenvolvimento Sustentável. [acessado 2010 jul]. Disponível em: http://new. paho.org/bra/index.php?option=com_content\& task $=$ view \&id $=397 \&$ Itemid $=539$

10. Barros MBA, César CLG, Carandina L, Torre GD. Desigualdades sociais na prevalência de doenças crônicas no Brasil: PNAD-2003. Cien Saude Colet 2006; 11(4):911-926.

11. Vargas CM, Burt VL, Gillum RF, Pamuk ER. Validity of self-reported hipertension in the national Health and Nutrition Examination Survey III. Prev Med 1997; 26:678-85.

12. Lima-Costa MF, Peixoto SV, Firmo JOA. Validade da hipertensão arterial auto-referida e seus determinantes (projeto Bambuí). Rev de Saúde Pub 2004; 38(5):637-642.

13. Alonso A, Beunza JJ, Delgado-Rodríguez M, Martínez-González MA. Validation of self reported diagnosis of hypertension in a cohort of university graduates in Spain. BMC Public Health 2005; 5(94):1-7.

14. Instituto Brasileiro de Geografia e Estatística (IBGE). Pesquisa Nacional por Amostra de Domicílios 2008: Notas metodológicas pesquisa básica, pesquisa especial de tabagismo e pesquisas suplementares de saúde e acesso à internet e posse de telefone móvel celular para uso pessoal. Rio de Janeiro: IBGE; 2009. [acessado 2010 abr]. Disponível em: <http://www.ibge.gov.br>.

15. Silva PLN, Pessoa DGC, Lila MF. Análise Estatística de Dados da PNAD: Incorporando a Estrutura do Plano Amostral. Cien Saude Colet 2002; 7(4):659670 . 
16. Lee ES, Forthofer RN, Lorimor RJ. Analyzing complex survey data. Beverly Hills: Sage Pubns; 1989. (Quantitative applications in the social sciences, n. 07-071).

17. Statistical Package for Social Sciencias (SPSS). [computer program]. Version 17.0. Chicago (Estados Unidos): SPSS Inc.; 2008.

18. Szwarcwald CL, Damacena GN. Amostras complexas em inquéritos populacionais: planejamento e implicações na análise estatística dos dados. Rev Bras Epidemiol 2008; 11(Supl. 1):38-45.

19. Mendoza-Sassi R, Béria JU. Utilización de los servicios de salud: una revisión sistemática sobre los factores relacionados. Cad Saude Publica 2001; 17(4): 819-832.

20. Rauber C. Disease management can be good for what ails patients and insurers. Mod Healthe 1999; 29(13):48-54.

21. Verbrugge LM. The Twain meet: empirial explanations of sex differences in health and mortality. $J$ Health Soc Behav 1989; 30(3):282-304.

22. Verbrugge LM, Wingard DL. Sex differentials in health and mortality. Women \& Health 1987; 12(2): 103-145.

23. Macintyre S, Ford G, Hund K. Do women "overreport" morbidity? Men's and women's responses to structured prompting on a standard question on long standing illness. Social Science and Medicine 1999; 48:89-98.

24. Frederick I. Health in rural Tanzania: the determinants of Health Status, Health Care Demand and Health care Choice. Leuven: Discussion Paper, Katholieke Univ. Leuven; 1998.

25. Costa JSD, Reis MC, Silveira Filho CV, Linhares RS, Piccinini F. Prevalência de consultas médicas e fatores associados, Pelotas (RS), 1999-2000. Rev Saude Publica 2008; 42(6):1074-1084.

26. Mendoza-Sassi R, Béria JU, Barros AJD. Outpatient health service utilization and associated factors: a population-based study. Rev Saúde Pub 2003; 37(3): 372-378.

27. Lima-Costa MF, Barreto SM, Giatti L. Condições de saúde, capacidade funcional, uso de serviços de saúde e gastos com medicamentos da população idosa brasileira: um estudo descritivo baseado na Pesquisa Nacional por Amostras de Domicílios. Cad Saúde Publica 2003; 19(3):735-743.

28. Capilheira MF, Santos IS. Fatores individuais associados à utilização de consultas médicas por adultos. Rev Saúde Pub 2006; 40(3):436-43.

29. Costa JSD, Facchini LA. Utilização de serviços ambulatoriais em Pelotas: onde a população consulta e com que freqüência. Rev Saúde Pub 1997; 31(4): 360-369.

30. Bahia L, Costa AJL, Fernandes C, Luiz RR, Cavalcanti MLT. Segmentação da demanda dos planos e seguros privados de saúde: uma análise das informações da PNAD/98. Cien Saude Colet 2002; 7(4): 671-686.
31. Travassos C, Viacava F, Pinheiro R, Brito A. Utilização dos serviços de saúde no Brasil: gênero, características familiares e condição social. $\mathrm{Rev} P a-$ nam Salud Publica 2002; 11(5/6):365-73.

32. Noronha KVMS, Andrade MV. Desigualdades sociais em saúde e na utilização dos serviços de saúde entre os idosos na América Latina. Rev Panam Salud Publica 2005; 17(5/6):410-418.

33. Bahia L, Luiz RR, Salm C, Costa AJL, Kale PL, Cavalcanti MLT. O mercado de planos e seguros de saúde no Brasil: uma abordagem exploratória sobre a estratificação das demandas segundo a PNAD 2003. Cien Saude Colet 2006; 11(4):951-965.

34. Viacava F, Dachs N, Travassos C. Os inquéritos domiciliares e o Sistema Nacional de Informações em Saúde. Cien Saude Colet 2006; 11(4):863-869.

35. Knigth M, Stewart-Brown S, Fletcher L. Estimating health needs: the impact of a checklist of conditions and quality of life measurement on health information derived from community surveys. $J$ Public Health 2001; 23(3):179-186.

36. Wu SC, Li CY, Ke DS. The agreement between selfreporting and clinical diagnosis for selected medical conditions among elderly in Taiwan. Public Health 2000; 114(2):137-142.

37. Hartshorn TA. Interpreting the city: an urban geography. 2a ed. [S. 1.]: Hohn Wiley \& Sons, Inc; 1992.

38. Kinra S. Comentary: Beyond urban-rural comparisons: towards a life course approach to understanding health effects of urbanization. Int J Epidemiol 2004; 33(4):777-778.

39. Kassouf AL. Acesso aos Serviços de Saúde nas Áreas Urbana e Rural do Brasil. Rev Econ Sociol Rural 2005; 43(1):29-44.

40. Luiz RR, Cabral MDB. Sensitivity analysis for an unmeasured confounder: a review of two independent methods. Rev Bras Epidemiol 2010; 13(2):188198.

41. Szklo M. The evaluation of epidemiologic evidence for policy-making. Am J Epidemiol 2001; 154(12) Supl.:S13-S17.

Artigo apresentado em 21/10/2010

Aprovado em 16/02/2011

Versão final aprovada em 25/02/2011 